\title{
Thyroid heterogeneity, as indicated by the CV of ultrasonographic intensities, correlates with anti-thyroid peroxidase antibodies in euthyroid Hashimoto's thyroiditis
}

Yosuke Wakita, Toshiki Nagasaki, Yuki Nagata, Yasuo Imanishi, Shinsuke Yamada, Koichiro Yoda, Masanori Emoto, Eiji Ishimura and Masaaki Inaba

\begin{abstract}
Objective: To prospectively evaluate the heterogeneous appearance of the thyroid gland, reflecting inflammation and destruction in euthyroid Hashimoto's thyroiditis (HT), we investigated the clinical utilities of the heterogeneity index (HI) [the coefficient of variance (CV) of the ultrasonographic (US) intensities], focusing on anti-thyroid peroxidase antibodies (TPO-Ab), which represent not only disease activity but also subsequent thyroid destruction of HT.

Methods: Forty-four consecutive patients with euthyroid HT [60.5 \pm 2.7 years old (mean \pm SE)] and 30 age-matched normal controls were studied. HI was calculated as the CV (SD/mean) of US intensities of either four points per lobe of the thyroid gland along a horizontal line at the depth of the right common carotid artery. Evaluation included serum levels of free thyroxine (FT4), free triiodothyronine (FT3), thyroid stimulating hormone (TSH), anti-thyroid peroxidase antibodies (TPO-Ab), anti-thyroglobulin antibodies (Tg-Ab), thyroglobulin and thyroid volume.

Results: While no differences were observed for $\mathrm{TSH}_{1} \mathrm{FT}_{4}$ and $\mathrm{FT}_{3}$, thyroglobulin and thyroid volume between the two groups, $\mathrm{HI}$ exhibited a tendency towards a significant difference $(3.59 \pm 0.20 \%$ in $\mathrm{HT}$ patients vs $3.23 \pm 0.19 \%$ in normal group, $p=0.089)$. In HT patients, there was a significant and positive correlation of HI with TPO-Ab $(r=0.396, p=0.034)$, whereas such a correlation was absent in normal controls. In both groups, there were no significant correlations of $\mathrm{HI}$ with $\mathrm{Tg}-\mathrm{Ab}, \mathrm{FT}_{3}, \mathrm{FT}_{4}$ or TSH.

Conclusions: This is the first report of the close relation between heterogeneity of US of the thyroid gland and TPO-Ab in euthyroid HT patients before the heterogeneity becomes distinguishable from normal thyroid glands. Furthermore, at this stage, subsequent thyroid destruction in HT might be already be predicted through the heterogeniety of the thyroid tissue.
\end{abstract}

Keywords: Hashimoto's thyroiditis, Heterogeneity, Ultrasonography, Anti-thyroid peroxidase antibodies

\section{Introduction}

Ultrasonographic (US) examination is a very accurate and highly sensitive method for assessing thyroid gland lesions, especially thyroid nodules, that can be performed quickly even in out-patient clinics [1]. Nevertheless, few studies have reported the use of US to estimate inflammation and destruction of thyroid parenchymal tissue. While

\footnotetext{
* Correspondence: toshi-n@jg7.so-net.ne.jp

Department of Metabolism, Endocrinology and Molecular Medicine, Osaka City University Graduate School of Medicine, 1-4-3, Asahi-machi, Abeno-ku, Osaka-city 545-8585, Japan
}

hypoechogenicity has been reported in untreated and levothyroxine-treated overt hypothyroid Hashimoto's thyroiditis (HT) patients [2,3], the clinical utility of measuring US intensities remains unknown in euthyroid HT without apparent hypoechogenicity.

In the current report, we used a newly-designed sensitive US method that was able to distinguish from the hypoechogenicity $[2,3]$ and enabled to assess and quantify heterogeneity in the euthyroid state, as the heterogeneity index (HI) [CV of the US intensities of eight points of thyroid gland on horizontal line between bilateral common

\section{C) Biomed Central}


carotid arteries]. This method was not affected by depth of the thyroid gland and weakening of ultrasound because it was based on the CV (SD/mean) of the US intensities along a horizontal line.

For patients with HT, a highly sensitive radioimmunoassay system for anti-thyroid antibodies [anti-thyroglobulin antibodies (Tg-Ab) and anti-thyroid peroxidase antibodies (TPO-Ab)] has proven to be essential for diagnosis [4]. In particular, TPO-Ab has been established to represent the current activity of HT and subsequent thyroid destruction, because the TPO-antigen is closely involved with cellmediated cytotoxicity, whereas the Tg-Ab is not [5].

Therefore, we investigated the clinical meaning of $\mathrm{HI}$ in euthyroid HT patients, and attempted to correlate HI with a variety of thyroid markers, such as thyroid hormones, thyroglobulin and thyroid volume, and autoimmune anti-thyroid antibodies.

\section{Patients and methods Subjects}

The study was approved by the ethical review committee of Osaka City University Hospital. Written informed consent was obtained from each patient. Newly detected $56 \mathrm{HT}$ patients in a euthyroid state $(\mathrm{M} / \mathrm{F}, 10 / 40)$ were enrolled consecutively during the 7 -month period from January to July, 2011.

The diagnosis of HT was made based on the elevation of either $\mathrm{Tg}-\mathrm{Ab}$ or TPO-Ab above the normal upper limit. To confirm sustained euthyroidism, thus excluding patients with a temporary condition, i.e., during the recovery from painless thyroiditis or occult subacute thyroiditis, measurement of TSH was conducted at least twice over 6 months. Moreover, to avoid confounding factors known to affect thyroid hormones, patients who were pregnant or post-partum [6], patients receiving other hormone-replacement therapy or taking any drugs that could affect thyroid hormones, such as amiodarone hydrochloride, interferon and Interleukin-2, lithium carbonate, gonadotropin releasing hormone, raloxifene hydrochloride, anti-convulsion drugs, and rifampicin, and those with autoimmune antibodies, were excluded from the study $[7,8]$.

Normal control subjects who joined the Health-Check Program at Osaka City University Hospital were enrolled consecutively as age-matched controls (M/F, 10/40). Normal controls had no history of thyroid disease, had neither goiter nor an autoimmune-antibody titer, and were in a euthyroid state.

The smoking index (the daily number of cigarettes multiplied by the number of years of smoking) was similar between the HT patients and normal controls and individual smoking habits did not change during this study.

\section{Serum parameters}

Blood was drawn just before ultrasonography after overnight fasting. Commercially available electrochemiluminescent immunoassay (ECLIA) kits were used for the thyroid hormone assay. TSH was measured by sandwich Elecsys $^{\circledR}$, and FT4 and FT3 levels were determined by competitive Elecsys ${ }^{\circledR}$ (Roche Diagnostics K.K., Tokyo, Japan) [9]. Anti-thyroglobulin (Cosmic Co., Tokyo, Japan) and anti-thyroid peroxidase antibodies (Cosmic Co., Tokyo, Japan) were also determined using highly-sensitive, commercially available, radioimmunoassay kits [10]. In this system, highly purified thyroglobulin and thyroid peroxidase were used as antigens. Serum concentrations of anti-thyroglobulin and anti-thyroid peroxidase antibodies greater than 0.4 and $0.3 \mathrm{U} / \mathrm{ml}$, respectively, were considered positive. Serum thyroglobulin levels were measured using an ECLIA kit, Elecsys ${ }^{\circledR}$ (Roche Diagnostics K.K., Tokyo, Japan) [11].

\section{Quantitative ultrasonographic measurements of heterogeneity index and thyroid volume}

Thyroid HI was determined using an US apparatus (LOGIQ 7PRO, GE healthcare, Princeton, NJ, USA) with a $10-\mathrm{MHz}$ linear array probe operating in B-mode (Figure 1). Four open-circle cursors (2 $\mathrm{mm}$ in diameter) per half lobe were positioned along a horizontal line at the depth of the right common carotid artery adjacent to the thyroid gland. US intensity within each circle was automatically calculated immediately after one cursor was set. HI was defined as the $\mathrm{CV}$ (SD/mean) of eight US intensities. All measurements were performed by the same examiner (Y.N.), who was blinded to the characteristics of the subjects.

Thyroid volume was also determined by ultrasonography, based on a calculation using an ellipsoid model (width $\times$ length $\times$ thickness $\times 0.7$ for each lobe) [12]

\section{Statistical analysis}

Data are expressed as the mean \pm SE unless otherwise indicated. Statistical analyses were performed using StatView (version 5.0, SAS Institute, Cary, NC). Differences in clinical factors between HT patients and normal controls were examined using the Mann-Whitney $U$ test for assessment of the median values. The difference in the male/female ratio between the two groups was analyzed using the chi-square test. Simple regression analysis was used to examine the relationships between the two factors. $P$-values less than 0.05 were considered to be statistically significant.

\section{Results}

Clinical variables in normal controls and HT patients

A comparison of the variables between normal controls and HT patients is presented in Table 1. In addition to 

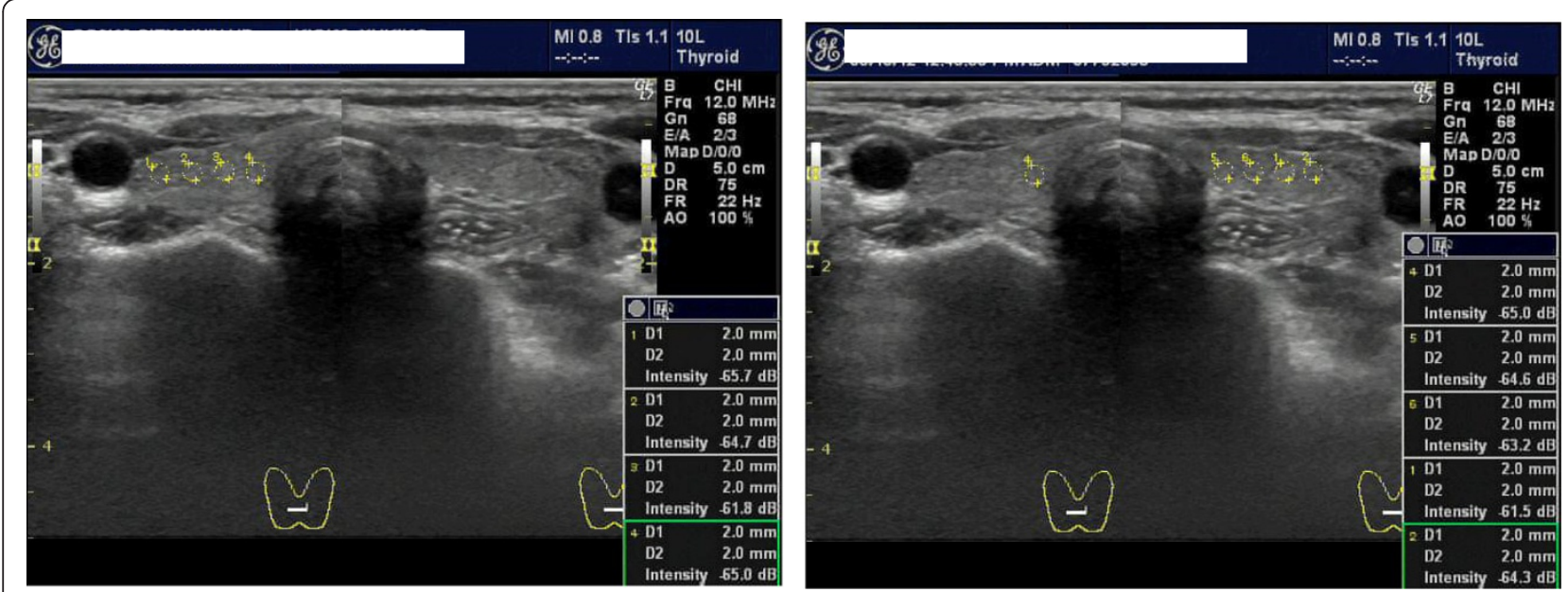

Figure 1 Recording of the ultrasonographic intensities and calculation of the thyroid heterogeneity index (HI). The appropriate sampling points were first determined for measuring. Four open-circle cursors (2 $\mathrm{mm}$ in diameter) per half lobe were positioned straight along a horizontal line at the depth of the right common carotid artery adjacent to the thyroid gland. US intensity within each circle was automatically calculated immediately after the cursor was set. HI was defined as the CV (i.e., SD/mean) of eight US intensities.

common biochemical markers, such as liver enzymes, renal function and lipid profiles, no significant differences were found in age, male/female ratio, BMI, smoking index, the serum level of $\mathrm{TSH}, \mathrm{FT}_{3}$ and $\mathrm{FT}_{4}$, thyroglobulin, or thyroid volume between normal controls and HT patients (data not shown).

\section{$\mathrm{HI}$ in normal controls and $\mathrm{HT}$ patients}

As shown in Table 1, HI exhibited a tendency towards a significant difference between normal controls and HT patients $(3.23 \pm 0.19 \%$ in the normal group vs. $3.59 \pm 0.20 \%$ in the HT patients, $\mathrm{p}=0.089$ ).

\section{Correlations between $\mathrm{HI}$ and clinical variables in normal} controls and $\mathrm{HT}$ patients

The correlations between $\mathrm{HI}$ and the clinical variables in normal controls and HT patients are presented in Table 2. In the HT patients there was a significant and positive correlation between $\mathrm{HI}$ and TPO-Ab $(\mathrm{r}=0.396$, $\mathrm{p}=0.034)$ but not Tg-Ab $(\mathrm{r}=-0.014, \mathrm{p}=0.941)$ or TSH $(\mathrm{r}=-0.012, \mathrm{p}=0.953)$. In normal controls $\mathrm{HI}$ did not correlate with these factors. There were no significant correlations between $\mathrm{HI}$ and age, BMI, smoking index, serum level of $\mathrm{FT}_{3}, \mathrm{FT}_{4}$, thyroglobulin or thyroid volume in either group (data not shown).

\section{Table 1 Baseline characteristics of the subjects with Hashimoto's thyroiditis and the controls}

\begin{tabular}{|c|c|c|c|}
\hline & Subjects with Hashimoto's thyroiditis & Normal controls & $\mathbf{P}$ \\
\hline Number of subjects & 44 & 44 & \\
\hline Gender (female/male) & $32 / 12$ & $32 / 12$ & ns \\
\hline Age (years) & $60.5 \pm 2.7$ & $58.7 \pm 1.9$ & ns \\
\hline Body mass index $\left(\mathrm{kg} / \mathrm{m}^{2}\right)$ & $21.7 \pm 0.48$ & $21.4 \pm 0.58$ & ns \\
\hline Smoking index & $74 \pm 59$ & $67 \pm 72$ & ns \\
\hline $\operatorname{Tg}-\mathrm{Ab}(\mathrm{U} / \mathrm{ml})[<0.4]$ & $8.37 \pm 2.55$ & $<0.4$ & \\
\hline $\mathrm{TPO}-\mathrm{Ab}(\mathrm{U} / \mathrm{ml})[<0.3]$ & $15.3 \pm 6.13$ & $<0.3$ & \\
\hline $\mathrm{FT}_{4}(\mathrm{pmol} / \mathrm{l})$ [11.6-21.9] & $14.5 \pm 0.37$ & $14.6 \pm 0.57$ & ns \\
\hline $\mathrm{FT}_{3}(\mathrm{pmol} / \mathrm{l})[3.54-6.16]$ & $4.17 \pm 0.11$ & $3.95 \pm 0.15$ & ns \\
\hline $\mathrm{TSH}(\mathrm{mIU} / \mathrm{l})[0.5-5]$ & $2.40 \pm 0.33$ & $2.53 \pm 0.28$ & ns \\
\hline Thyroglobulin (mg/l) [0.0-30.0] & $43.4 \pm 20.6$ & $40.6 \pm 11.5$ & ns \\
\hline Thyroid volume $\left(\mathrm{mm}^{3}\right)$ & $14687.1 \pm 1494.3$ & $15757.2 \pm 1984.4$ & ns \\
\hline $\mathrm{HI}(\%)$ & $3.23 \pm 0.19$ & $3.59 \pm 0.20$ & $\mathrm{~ns}(0.089)$ \\
\hline
\end{tabular}

Data are expressed as the mean \pm SE. The differences between two groups were examined using the Mann-Whitney $U$ test for assessment of the medians. $n s$ : not significant, Systolic BP: systolic blood pressure, Diastolic BP: diastolic blood pressure, $T g$ - $A b$; anti-thyroglobulin antibody, TPO-Ab; antithyroid peroxidase antibody, $\mathrm{Hl}$ : heterogeneity index. 
Table 2 Correlations between the heterogeneity index and other parameters in subjects with Hashimoto's thyroiditis and controls

\begin{tabular}{|c|c|c|c|c|}
\hline & \multicolumn{2}{|c|}{$\begin{array}{l}\text { Subjects with Hashimoto's } \\
\text { thyroiditis }\end{array}$} & \multicolumn{2}{|c|}{$\begin{array}{l}\text { Normal } \\
\text { controls }\end{array}$} \\
\hline & $r$ & $\mathbf{P}$ & $r$ & $\mathbf{P}$ \\
\hline Age (years) & 0.077 & ns & 0.082 & ns \\
\hline Body mass index & 0.253 & ns & 0.201 & ns \\
\hline Smoking index & 0.292 & ns & 0.201 & ns \\
\hline Systolic BP & 0.044 & ns & 0.153 & ns \\
\hline Diastolic BP & -0.045 & ns & 0.279 & ns \\
\hline Pulse rate & -0.143 & ns & -0.017 & ns \\
\hline Tg-Ab & -0.014 & ns & & \\
\hline TPO-Ab & 0.396 & 0.034 & & \\
\hline $\mathrm{FT}_{4}$ & -0.045 & ns & 0.188 & ns \\
\hline $\mathrm{FT}_{3}$ & -0.239 & ns & -0.048 & ns \\
\hline TSH & -0.125 & ns & -0.172 & ns \\
\hline Thyroglobulin & -0175 & ns & -0.204 & ns \\
\hline Thyroid volume & 0.264 & ns & 0.175 & ns \\
\hline
\end{tabular}

Data are expressed as the mean \pm SE. Simple regression analysis was used. ns: not significant, Systolic BP: systolic blood pressure, Diastolic BP: diastolic blood pressure, $T g-A b$; anti-thyroglobulin antibody, $T P O-A b$; antithyroid peroxidase antibody, HI: heterogeneity index.
Figure 2 presents the correlations between $\mathrm{HI}$ and $\mathrm{TPO}-\mathrm{Ab}, \mathrm{Tg}-\mathrm{Ab}$ and TSH in the HT patients.

\section{Discussion}

The results of the study revealed a significant and positive correlation between $\mathrm{HI}$ and TPO-Ab in euthyroid HT patients. In these patients, HI might reflect not only the extent of current inflammation and destruction of the thyroid tissue, but also subsequent destruction in HT, because $\mathrm{TPO}-\mathrm{Ab}$ is responsible for these changes through an autoimmune reaction in HT [5]. However, in the cases with very mild destruction, this correlation likely suggests the subsequent potential for terminal destruction.

TPO, originally described as thyroid microsomal antigen, is present on the apical surface of thyroid follicular cells and is the antigen most closely involved in cell-mediated cytotoxicity [13]. Hence, among auto-immune antibodies specific for the thyroid gland, the TPO-Ab titer represents the degree of lymphocytic infiltration of the thyroid gland, reflecting the current activity of $\mathrm{HT}$, as well as subsequent stages in the development of HT [5,13].

Nevertheless, since only a tendency of HI was found between HT patients and normal controls (Table 1), inflammation and destruction of the thyroid tissue would likely be slight or mild in the early phase of HT, namely the euthyroid state. Therefore, the clinical interpretation of
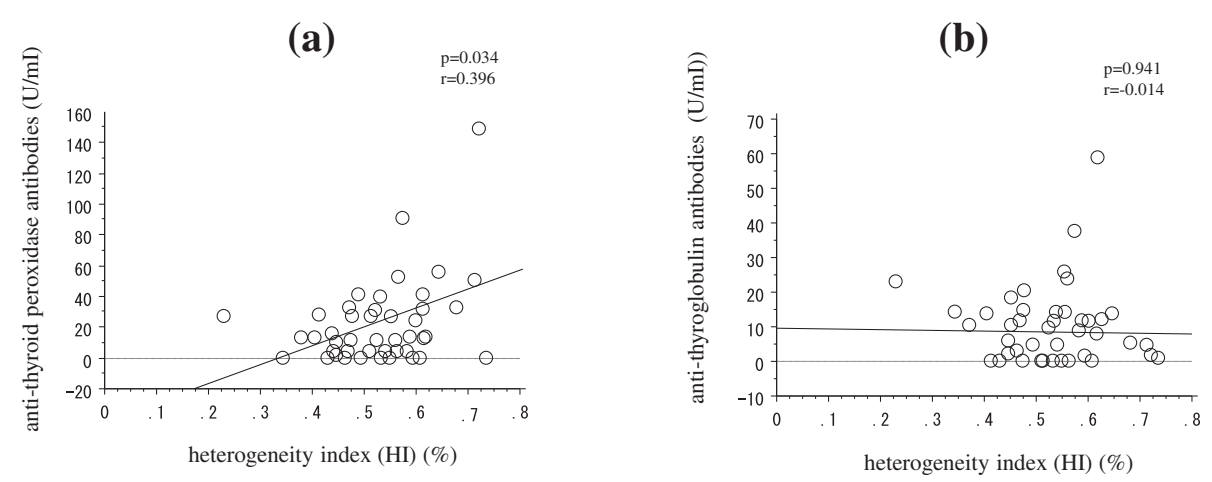

(c)

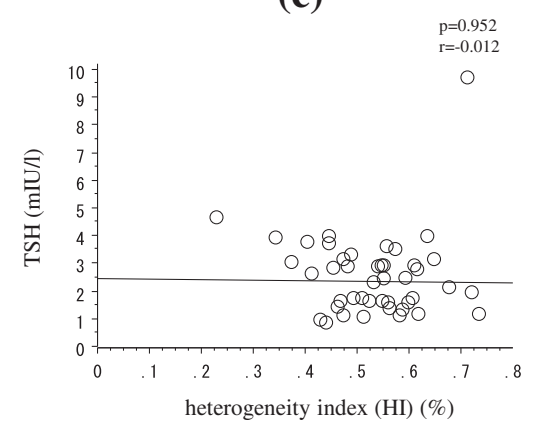

Figure 2 Simple regression analysis to determine the correlation of the heterogeneity index (HI) with anti-thyroid peroxidase antibody (TPO-Ab) (a), anti-thyroglobulin antibody (Tg-Ab) (b), and TSH (c) in Hashimoto's thyroiditis (HT). HI correlated significantly with TPO-Ab $(r=0.396, p=0.034)$, but not with $\mathrm{Hl}$ and Tg-Ab or TSH $(r=-0.014, p=0.941$ and $r=-0.012, p=0.953$, respectively). 
HI might be not the degree of current inflammation and destruction but more likely a measure of the potential for terminal destruction of the thyroid gland that can be achieved in the early stages of HT.

In a previous report, an interaction between the HLADRB4 and cytotoxic T-lymphocyte associated antigen 4 (CTLA-4) genes was shown to determine the thyroid function of TPO-positive Japanese HT patients with goiter [13]. This is consistent with our findings, supporting that terminal destruction of thyroid gland is genetically predisposed.

Meanwhile, $\mathrm{Tg}-\mathrm{Ab}$, another auto-immune anti-thyroid antibody [4], did not correlate with HI in HT patients (Figure 2). Multiple antigen configurations of thyroglobulin are produced when it becomes iodinated, resulting in functionally active but immunologically distinct molecules [13]. Therefore, changes in $\mathrm{Tg}$ - $\mathrm{Ab}$ could occur independent of immune responses or the consequent inflammation and destruction in HT.

The lack of correlation of $\mathrm{HI}$ with $\mathrm{FT}_{3}, \mathrm{FT}_{4}$ and $\mathrm{TSH}$ in HT patients (Table 2) might be explained by similar degrees of heterogeneity and thyroid hormones between HT patients and normal controls. Thus, in the euthyroid state of early HT inflammation, the simultaneous destruction of thyroid tissue had not progressed sufficiently to affect these factors. If measured in a stage of overt hypothyroidism, a correlation might be observed between $\mathrm{HI}$ and thyroid hormones, as was reported previously, with respect to hypoechogenicity in HT $[2,3]$. This is the subject of ongoing and future investigations by our group.

Serum thyroglobulin did not correlate with $\mathrm{HI}$ in this study (Table 2). Possible explanations for this are as follows: although thyroglobulin is intimately associated with the thyroid, it is a normal component of the blood, as some thyroglobulin is invariably secreted, during normal thyroid hormone release [14]. Therefore, the elevation of serum thyroglobulin in HT could be due to increased leak in response to TSH stimulation or the excessive production by the associated cancer. Moreover, problems include variable sensitivity and reproducibility of the assays and interference by thyroglobulin autoantibodies [15]. Hence, in the euthyroid state the existence of $\mathrm{Tg}$-Ab thyroglobulin may be of less clinical importance.

HI did not correlate with thyroid volume (Table 2), likely due to the same reason of the early stage of HT; i.e., in the presence of a very mild inflammation and early destruction of thyroid tissue in the euthyroid state of $\mathrm{HT}$, the changes in thyroid volume did not reach statistical significance. In another previous investigation of overt autoimmune hypothyroidism, thyroid volume correlated negatively with echogenicity [16].

$\mathrm{HI}$ is easily and reproducibly obtained using ultrasonography, and is not affected by the depth of the thyroid gland and weakening of ultrasound, because it represents the $\mathrm{CV}$ (SD/mean) of US intensities along a horizontal line.

Thus, computerized grey-scale analysis using US was previously reported to be useful in quantifying thyroid hypoechogenicity in untreated and levothyroxine-treated overt hypothyroid HT patients [7]. The marked difference from our method is not in the assessment of heterogeneity but in the quantification of hypoechogenicity, which would be affected by the depth of the thyroid gland and weakening of the ultrasound. Furthermore, while their subjects included untreated and levothyroxine-treated overt hypothyroid HT patients, our method was applied to euthyroid and subclinical hypothyroid patients with visually indistinguishable heterogeneity.

Further, computerized grey-scale analysis allows for significant correlations between hypoechogenicity and indices of the thyroid gland, namely serum TSH, FT4 and TPO-Ab values. Interestingly, even in overt hypothyroidism echogenicity did not correlate with $\mathrm{Tg}$ - $\mathrm{Ab}$, but rather correlated with TPO-Ab, possibly due to the same underlying reasons noted above.

One limitation of $\mathrm{HI}$ is obstruction by the tumor or cyst along the horizontal line for sampling, which interferes with positioning of the four open-circle cursors for each half lobe. Another limitation occurs when the thyroid gland is too small or atrophic, and cursors cannot be positioned properly. Since each open-circle is $2 \mathrm{~mm}$ in diameter, a straight line more than $8 \mathrm{~mm}$ in length is needed for each lobe.

In summary, our results demonstrate the close relation between heterogeneity of the thyroid gland and TPO-Ab in euthyroid and subclinical hypothyroid patients with Hashimoto's thyroiditis. It was suggested that even in such hormonal states, without distinguishable inflammation or destruction of thyroid tissue compared with normal subjects, subsequent thyroid destruction of HT might already be indicated through heterogeniety of the thyroid tissue.

\section{Competing interests}

We declare that there is no competing interests that could be perceived as prejudicing the impartiality of the research reported.

\section{Authors' contributions}

YW carried out the ultrasonographic measurements and analyzed the data. TN drafted the protocol and manuscript, analyzed the data. YN assisted ultrasonographic measurements and analysis of the data. YI, SY, KY, ME, EI and $\mathrm{MI}$ participated in the logical composition of manuscript. All authors read and approved the final manuscript.

\section{Acknowledgement}

This research project was not supported by any specific grant from any funding agency in the public, commercial or not-for-profit sectors.

Received: 11 February 2013 Accepted: 12 March 2013

Published: 23 March 2013 


\section{References}

1. Ross DS: Nonpalpable thyroid nodules: managing an epidemic. J Clin Endocrinol Metab 2002, 87:1938-1940.

2. Mazziotti G, Sorvillo F, lorio S, Carbone A, Romeo A, Piscopo M, Capuano S, Capuano E, Amato G, Carella C: Grey-scale analysis allows a quantitative evaluation of thyroid echogenicity in the patients with Hashimoto's thyroiditis. Clin Endocrinol (Oxf) 2003, 59:223-229.

3. Schiemann U, Avenhaus W, Konturek JW, Gellner R, Hengst K, Gross M: Relationship of clinical features and laboratory parameters to thyroid echogenicity measured by standardized grey scale ultrasonography in patients with Hashimoto's thyroiditis. Med Sci Monit 2003, 9:13-17.

4. Engler H, Riesen WF, Keller B: Anti-thyroid peroxidase (anti-TPO) antibodies in thyroid diseases, non-thyroidal illness and controls. Clinical validity of a new commercial method for detection of anti-TPO (thyroid microsomal) autoantibodies. Clin Chim Acta 1994, 225:123-136.

5. Sinclair D: Clinical and laboratory aspects of thyroid autoantibodies. Ann Clin Biochem 2006, 43:173-183.

6. Chiovato L, Lapi P, Fiore E, Tonacchera M, Pinchera A: Thyroid autoimmunity and female gender. J Endocrinol Investig 1993, 16:384-391.

7. Bindra A, Braunstein GD: Thyroiditis. Am Fam Physician 2006, 15:1769-1776.

8. Kasayama S, Miyake S, Samejima Y: Transient thyrotoxicosis and hypothyroidism following administration of the $\mathrm{GnRH}$ agonist leuprolide acetate. Endocr J 2000, 47:83-785.

9. Kulmala S, Suomi J: Current status of modernanalytical luminescence methods. Anal Chim Acta 2003, 500:21-69.

10. Arai T, Kurashima C, Utsuyama M, Sawabe M, Ito H: Measurement of antithyroglobulin and anti-thyroid peroxidase antibodies using highly sensitive radioimmunoassay: an effective method for detecting asymptomatic focal lymphocytic thyroiditis in the elderly. Endocr J 2000, 47:575-582.

11. Algeciras-Schimnich A, Lasho MA, Ness KM, Cheryk LA, Grebe SK: The Roche elecsys and Siemens-centaur thyroglobulin autoantibody assays show comparable clinical performance to the recently unavailable Beckmancoulter access thyroglobulin autoantibody assay in identifying samples with potentially false-low thyroglobulin measurements due to thyroglobulin autoantibody interference. Thyroid 2011, 21:813-814.

12. Urakami Y, Takamatsu J, Sakane S, Kuma K, Ohsawa N: Changes in thyroid volume in response to radioactive iodine for Graves' hyperthyroidism correlated with activity of thyroid-stimulating antibody and treatment outcome. J Clin Endocrinol Metab 1996, 81:3257-3260.

13. Terauchi M, Yanagawa T, Ishikawa N, Ito K, Fukazawa T, Maruyama H, Saruta $\mathrm{T}$ : Interactions of HLA-DRB4 and CTLA-4 genes influence thyroid function in Hashimoto's thyroiditis in Japanese population. J Endocrinol Invest 2003, 26:1208-1212.

14. Lerman J: lodine components of the blood. Circulating thyroglobulin in normal persons and in persons with thyroid disease. J Clin Invest 1940, 19:555-560

15. Pacini F, Pinchera A: Serum and tissue thyroglobulin measurement: clinical applications in thyroid disease. Biochimie 1999, 81:463-467.

16. Carlé A, Pedersen IB, Knudsen N, Perrild H, Ovesen L, Jørgensen T, Laurberg $P$ : Thyroid volume in hypothyroidism due to autoimmune disease follows a unimodal distribution: evidence against primary thyroid atrophy and autoimmune thyroiditis being distinct diseases. $J$ Clin Endocrinol Metab 2009, 94:833-839.

doi:10.1186/1756-6614-6-5

Cite this article as: Wakita et al:: Thyroid heterogeneity, as indicated by the CV of ultrasonographic intensities, correlates with anti-thyroid peroxidase antibodies in euthyroid Hashimoto's thyroiditis. Thyroid Research 2013 6:5.

\section{Submit your next manuscript to BioMed Central and take full advantage of:}

- Convenient online submission

- Thorough peer review

- No space constraints or color figure charges

- Immediate publication on acceptance

- Inclusion in PubMed, CAS, Scopus and Google Scholar

- Research which is freely available for redistribution

Submit your manuscript at www.biomedcentral.com/submit
C BioMed Central 